\title{
Investigação do Seis Sigma na redução do tempo de setup: uma pesquisa empírica em uma empresa do setor de polímeros
}

\author{
Oliver de Góes Corrêa \\ Universidade Federal de São Carlos - Campus Sorocaba \\ olivergoes@terra.com.br
}

\author{
Nara Rossetti \\ Universidade Federal de São Carlos - Campus Sorocaba \\ nara@ufscar.br

\section{Ricardo Coser Mergulhão} \\ Universidade Federal de São Carlos - Campus Sorocaba \\ mergulhao@ufscar.br \\ Jorge Luis Faria Meirelles \\ Universidade Federal de São Carlos - Campus Sorocaba \\ jorgeluis@ufscar.br

\section{Brena Bezerra Silva} \\ Universidade Federal de São Carlos - Campus Sorocaba \\ bezerra.brena@gmail.com
}

\section{RESUMO}

Este artigo investiga a aplicação do método DMAIC do Seis Sigma combinado com a técnica SMED (single minute exchange of dies, ou aproximando para o português, troca rápida de ferramenta) na redução do tempo de setup em uma multinacional do setor de polímeros. O método de pesquisa utilizado no desenvolvimento do artigo foi o da pesquisa-ação. Os resultados desta pesquisa indicam que o Seis Sigma no âmbito da aplicação do DMAIC possibilita que a SMED seja desenvolvida de forma mais estruturada e eficiente devido ao apoio dos belts e demais ferramentas e métodos do Seis Sigma. Além disso, no desenvolvimento do projeto Seis Sigma foram identificados fatores que influenciam no desenvolvimento dessa integração, são eles: mudança cultural, educação e treinamento contínuos, e compreensão do programa Seis Sigma.

Palavras-chave: Seis Sigma. DMAIC. SMED.

\begin{abstract}
The purpose of this paper is to investigate the integration of Six Sigma DMAIC method and SMED (single minute exchange of dies) method in a project to reduce setup time in a multinational industry of polymers. The research method employed was action research. The results suggest that Six Sigma under DMAIC method contributes to take the SMED method to more structured and efficient due the Six Sigma belts and tools support. Furthermore, in Six Sigma project, the identified factors that affecting the integration were: cultural change, continuous education and training, and understanding of Six Sigma program.
\end{abstract}

Keywords: Six Sigma. DMAIC. SMED. 


\section{Introdução}

Na tentativa de manter ou construir vantagem competitiva, abordagens e iniciativas estratégicas são adotadas por muitas empresas (YUSR et al., 2012). Exemplos dessas práticas incluem o Total Quality Management (TQM), Reengenharia de Processos, os critérios do Malcolm Baldrige Award, a certificação ISO 9001, o programa Seis Sigma e o Lean Manufacturing (BENNER; VELOSO, 2008). Dentre essas, duas que se destacam são o Seis Sigma e o Lean Manufacturing (ARNHEITER; MALEYEFF, 2005; SALAH et al., 2010).

O Seis Sigma objetiva reduzir a variabilidade dos processos e uma peculiaridade dessa abordagem está no uso do método DMAIC (Define, Measure, Analyse, Improve e Control), que direciona o desenvolvimento de projetos Seis Sigma no uso de métodos estatísticos e ferramentas da qualidade (CORONADO; ANTONY, 2002).

O Lean Manufacturing tem foco na redução dos desperdícios, entre eles, o tempo de espera (BEN NAYLOR et al., 1999). Uma técnica que pode ser utilizada no contexto do Lean é a SMED (Single Minute Exchange Die), que objetiva otimizar o tempo de setup dos equipamentos, promovendo maior produtividade e flexibilidade na linha de produção.

De acordo com Cheng e Chang (2012), o uso conjunto do Lean Manufacturing com o Seis Sigma pode melhorar a qualidade, reduzir a variância e eliminar os desperdícios. Os conceitos do Lean relacionados à eliminação de desperdícios (SHINGO, 2000) podem ser associados aos objetivos do Seis Sigma, como redução da variabilidade e aumento do desempenho financeiro (PANDE et al., 2000). Arnheiter e Maleyeff (2005) destacam que o Lean Manufacturing e o Seis Sigma podem, eventualmente, conduzir a retornos decrescentes devido à possível falta de entendimento do processo de integração entre essas abordagens. Ao lado disso, Salah et al. (2010) também destacam que estudos sobre a integração entre essas duas abordagens são relevantes na medida que estendem a compreensão do assunto.

Nesse contexto, torna-se relevante investigar de como essas duas abordagens podem ser integradas no que diz respeito ao método DMAIC e à técnica SMED. Com isso, esse artigo objetiva, por meio de uma pesquisa-ação, investigar a aplicação do DMAIC com a SMED, no contexto de um projeto de melhoria para a redução do tempo de setup de um processo de conversão de filme de polipropileno em uma multinacional manufatureira.

A seguir apresenta-se uma breve revisão teórica sobre Seis Sigma e SMED. Após isso, a pesquisa de campo conjuntamente com as análises dos resultados. E, por último, são apresentadas as conclusões deste artigo.

\section{Seis Sigma}

O programa Seis Sigma é um sistema que busca reduzir a variação nos processos organizacionais, utilizando especialistas de melhoria, um método estruturado e métricas de desempenho com o objetivo de alcançar objetivos estratégicos (SCHROEDER et al., 2008).

Esse programa, de acordo com Braunscheidel et al. (2011), é uma das inovações mais recentes de gestão da qualidade que muitas organizações têm adotado com a intenção de melhorar significativamente o desempenho e atendimento ao cliente.

Em termos estatísticos o programa Seis Sigma difunde a métrica do sigma, que está associada ao desvio-padrão de uma população de modo que um processo Seis Sigma teria desempenho capaz de apresentar no máximo 3,4 defeitos por milhões de oportunidades.

O programa Seis Sigma não é uma proposta revolucionária, mas um programa de melhoria contínua que utiliza ferramentas, técnicas e abordagens anteriores, como o diagrama de Ishikawa que auxilia na descoberta das causas fundamentais de problemas no processo (FOLARON, 2003).

Uma distinção do programa Seis Sigma em relação a outros programas de melhoria é sua hierarquia de responsabilidades que é apresentada a seguir de acordo com o sistema Belt.

Champions e Sponsors - são indivíduos de nível hierárquico elevado na organização e que devem entender o Seis Sigma e se comprometerem com sua implantação e manutenção. São líderes empresariais treinados que promovem e levam a implantação do Seis Sigma em uma área significativa do negócio. Os Sponsors são líderes informais que fazem uso diário do Seis Sigma e procuram oportunidades de melhoria;

Master Black Belts - são pessoas da alta liderança técnica do programa e devem compreender as ferramentas da qualidade e os métodos estatísticos para auxiliarem os Black Belts na elaboração dos projetos de melhoria. A habilidade de comunicação e ensino é fundamental para o perfil deles;

Black Belts - são especialistas que dominam as ferramentas da qualidade e métodos estatísticos e que estão ativamente envolvidos no processo e acompanham todas as fases dos projetos Seis Sigma. Esses especialistas não só possuem habilidades técnicas, como também habilidades de liderança, desempenhando um papel essencial no Seis Sigma, porque são a ponte entre as equipes de gerência sênior e as equipes de melhoria do projeto; 
Green Belts - auxiliam os Black Belts na condução dos projetos Seis Sigma e também podem ser líderes de projetos menores, desde sua concepção até a conclusão. Costumam ter conhecimento substancial do processo com dedicação em tempo parcial. Além disso, eles costumam ser treinados por Master Black Belts e Black Belts (PINHO, 2005; SCHROEDER et al., 2008).

Dentro do contexto do Seis Sigma, os belts utilizam o método DMAIC para conduzir os projetos de melhoria durante suas cinco fases Define - Definir, Measure - Medir,
Analyse - Analisar, Improve - Melhorar, Control - Controlar. Esse método promove o uso do pensamento científico na medida em que estabelece um conjunto de etapas que estão logicamente relacionadas para buscar a solução de um problema.

No Quadro 1 são apresentadas as fases do DMAIC juntamente com os objetivos, principais atividades e as principais ferramentas da qualidade e métodos estatísticos usualmente utilizados.

Quadro 1 - Método de melhoria DMAIC do Seis Sigma.

\begin{tabular}{|c|c|c|c|}
\hline Fase & Objetivos & Principais Atividades & Ferramentas da qualidade e métodos estatísticos usuais \\
\hline $\begin{array}{l}\stackrel{\Xi}{E} \\
\stackrel{\Xi}{0}\end{array}$ & $\begin{array}{l}\text { Definir oportunidades e } \\
\text { fazer avaliação } \\
\text { financeira }\end{array}$ & $\begin{array}{l}\text { Definir requisitos do cliente; } \\
\text { Identificar variáveis de saída para medição do projeto; } \\
\text { Criar infraestrutura; } \\
\text { Avaliar impacto; e } \\
\text { Planejar projeto. }\end{array}$ & $\begin{array}{l}\text { Indicadores de desempenho } \\
\text { Custos da qualidade } \\
\text { Pesquisa de mercado } \\
\text { Quality Function Deployment } \\
\text { Diagrama de causa-e-efeito } \\
\text { Gráfico de Pareto } \\
\text { Mapa de raciocínio } \\
\text { Minuta do projeto }\end{array}$ \\
\hline 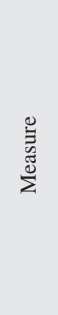 & $\begin{array}{l}\text { Mapear e mensurar os } \\
\text { processos }\end{array}$ & $\begin{array}{l}\text { Mapear processo; } \\
\text { Avaliar variáveis-chave de entrada; } \\
\text { Avaliar capabilidade do processo; } \\
\text { Identificar variáveis dominantes; e } \\
\text { Refinar problemas e objetivos. }\end{array}$ & $\begin{array}{l}\text { Fluxograma do processo; } \\
\text { Diagrama de causa-e-efeito; } \\
\text { Estatística descritiva; } \\
\text { MSA (Measure System Analyse); } \\
\text { FMEA (Failure Modes Effects and Analyse); } \\
\text { Planos de Controle; } \\
\text { Cartas de controle; } \\
\text { Capabilidade de processo; } \\
\text { Análise de Variância (ANOVA); } \\
\text { Boxplot; e } \\
\text { Séries temporais. }\end{array}$ \\
\hline 离 & $\begin{array}{l}\text { Analisar os dados e } \\
\text { identificar soluções }\end{array}$ & $\begin{array}{l}\text { Avaliar a relação entre variáveis de entrada e de saída; } \\
\text { Analisar a variação predominante; } \\
\text { Verificar a relação entre variáveis; e } \\
\text { Verificar e Implementar soluções. }\end{array}$ & $\begin{array}{l}\text { Matriz de causa-e efeito; } \\
\text { Estatística descritiva; } \\
\text { Boxplot; } \\
\text { Análise de regressão e correlação; } \\
\text { Intervalo de confiança; } \\
\text { Teste de hipóteses; } \\
\text { Análise de Variância (ANOVA); } \\
\text { Cartas multivariadas; } \\
\text { DOE (Design Of Experiments); e } \\
\text { Data Mining. }\end{array}$ \\
\hline 䚻 & $\begin{array}{l}\text { Aperfeiçoar processos e } \\
\text { obter resultados }\end{array}$ & $\begin{array}{l}\text { Selecionar fatores e níveis; } \\
\text { Executar experimentos e analisar resultados; } \\
\text { Determinar tolerâncias; e } \\
\text { Desenhar e implementar modificações no processo. }\end{array}$ & $\begin{array}{l}\text { DOE (Design Of Experiments); } \\
\text { Superfície de resposta; } \\
\text { OE (Operação evolutiva); e } \\
\text { Dimensionamento de tolerâncias. }\end{array}$ \\
\hline סे & $\begin{array}{l}\text { Padronizar e manter } \\
\text { ganhos obtidos }\end{array}$ & $\begin{array}{l}\text { Estabelecer padrões de medição para manter o } \\
\text { desempenho; e } \\
\text { Treinar os envolvidos. }\end{array}$ & $\begin{array}{l}\text { Plano de Controle; } \\
\text { Cartas de Controle; } \\
\text { Poka yoke; } \\
\text { Pré-controle; e } \\
\text { Instruções de trabalho. }\end{array}$ \\
\hline
\end{tabular}

Fonte: Adaptado de Pande et al. (2000).

A partir do Quadro 1 percebe-se a abrangência do DMAIC e os vários recursos que podem ser necessários para que um processo com desempenho Seis Sigma seja alcançado. Além disso, o DMAIC segue passos bem delineados para cada fase, como apresentado na coluna "Principais atividades”. Apesar da segmentação presente no Quadro 1 das ferramentas da qualidade e métodos estatísticos aplicados em cada fase, Satolo et al. (2009) destacam que uma ferramenta ou método não é estanque a determinada fase do DMAIC.
Os projetos Seis Sigma nem sempre obtém o resultado esperado pela organização e o índice de projetos que fracassam por custarem mais do que o estimado ou que excedem o tempo de execução previsto, é realmente alto (KWAK; ANBARI, 2004).

Convém destacar que existem fatores que precisam ser considerados durante a implementação e uso do Seis Sigma para que este programa tenha maior chance de atingir seus objetivos. Dentre eles, pode-se destacar: 
- O entendimento da alta administração sobre o Seis Sigma, uma vez que o comprometimento dela é essencial para que o apoio adequado seja fornecido ao programa (ARIENTE et al., 2005);

- O comprometimento dos indivíduos envolvidos no projeto e a presença de competências entre os utilizadores, tais como: conhecimento de ferramentas da qualidade e métodos estatísticos inerentes ao programa Seis Sigma; capacidade de mensurar custos e ganhos; e a comunicação durante a execução do projeto (RODRIGUES; WERNER, 2008);

- A mudança cultural é um fator envolvido com o ajuste nos valores, cultura, estrutura e infraestrutura da organização durante a implementação do Seis Sigma (ECKES, 2003).

- A presença de iniciativas prévias de qualidade também é um fator crítico de sucesso para a implantação do programa (TRAD; MAXIMIANO, 2009).

Antony e Banuelas (2002); Johnson e Swisher (2003) apresentam alguns fatores-chave para a implementação do Seis Sigma: compromisso e envolvimento da direção; compreensão do programa Seis Sigma, ferramentas e técnicas; seleção dos projetos estrategicamente mais importantes; infraestrutura organizacional; mudança de cultura; educação e treinamento contínuos para gerência e demais participantes; seleção cuidadosa dos líderes de projeto; e habilidade de administração de projetos.

Estudos recentes apontam alguns benefícios que o programa pode trazer para melhorar o desempenho das organizações (SWINK; JACOBS, 2012; SHAFER; MOLLER, 2012):

- O retorno sobre o investimento foi afetado positivamente com a adoção do Seis Sigma;

- O retorno sobre vendas melhorou com a adoção do Seis Sigma;

- O retorno sobre ativos melhorou positivamente;

- O lucro operacional sobre o ativo total e o lucro operacional sobre vendas melhoraram após o terceiro ano de implementação;

- Houve melhor taxa de melhoria no que diz respeito à produtividade;

- O desempenho do Seis Sigma tornou-se mais significativo a partir de dois anos de implementação.

Enfim, o desenvolvimento dos projetos Seis Sigma pelos belts mediante o método DMAIC parece fornecer uma estrutura adequada para a resolução de problemas e melhoria de processos, no entanto, existem fatores que precisam ser considerados, pois eles podem conduzir ao sucesso ou fracasso dos projetos e consequentemente do programa Seis Sigma.

\section{SMED}

A SMED (Single Minute Exchange Die) ou, em português, TRF (Troca Rápida de Ferramenta) é uma técnica que foi desenvolvida por Shigeo Shingo na década de 50 e visa reduzir sistematicamente o tempo de setup de ferramentas (McINTOSH et al., 2007; KARASU et al., 2014).

O tempo de setup (ou preparação) de máquina está presente em todos os tipos de operações e não agrega valor ao produto (SHINGO, 2000). A redução desse tempo conduz a um aumento da produtividade e da flexibilidade de uma linha produtiva e isso faz com que a SMED esteja alinhada aos princípios do Lean Manufacturing (CAKMAKCI, 2009).

Na teoria, a SMED envolve um conjunto de técnicas que possibilitam desempenhar o setup dos equipamentos e processos em menos de 10 minutos como meta (CAKMAKCI, 2009).

A SMED abrange três estágios, são eles segundo Shingo (2000, p. 50):

- Estágio 0 (ou estágio preliminar): Os tipos de setup (interno e externo) não são diferenciados. Este estágio tem como função a tomada de tempo das atividades do setup independente do momento em que ocorrem;

- Estágio 1: Separar setup interno e externo. Neste estágio ocorre um estudo em que são identificados os setups interno e externo durante o processo, sendo que o primeiro ocorre quando a máquina está parada em função da necessidade de setup, já o externo é oriundo de atividades realizadas enquanto a máquina está em funcionamento. Logo, o setup externo é realizado antes da contagem de tempo do setup interno;

- Estágio 2: Converter setup interno em externo. Somente realizar atividades possíveis durante o funcionamento da máquina não é suficiente para atingir o princípio da SMED, deve-se buscar oportunidades de converter setup interno em externo focando a meta de 10 minutos; e

- Estágio 3: Racionalizar todos os aspectos da operação de setup. Mesmo que o princípio da SMED seja trabalhar com tempos de setup abaixo de 10 minutos, isto nem sempre é verdade. Ou seja, cada caso deve ser analisado detalhadamente de maneira racional. E ainda, os estágios 2 e 3 não precisam, necessariamente, serem executados sequencialmente, podendo ser simultâneos.

Para consolidação da SMED, Shingo (2000) sugere a implementação de um check-list de setup, no qual são apresentados os passos e atividades de cada operador 
durante o processo de setup, facilitando e padronizando as atividades, de forma que seja possível que os operadores possam controlar a execução dessas atividades.
Na Figura 1 apresenta-se de maneira esquemática os estágios conceituais citados anteriormente e as principais técnicas associadas a cada estágio da SMED.

Figura 1 - Processo de SMED de Shingo: Estágios Conceituais e Técnicas Práticas.

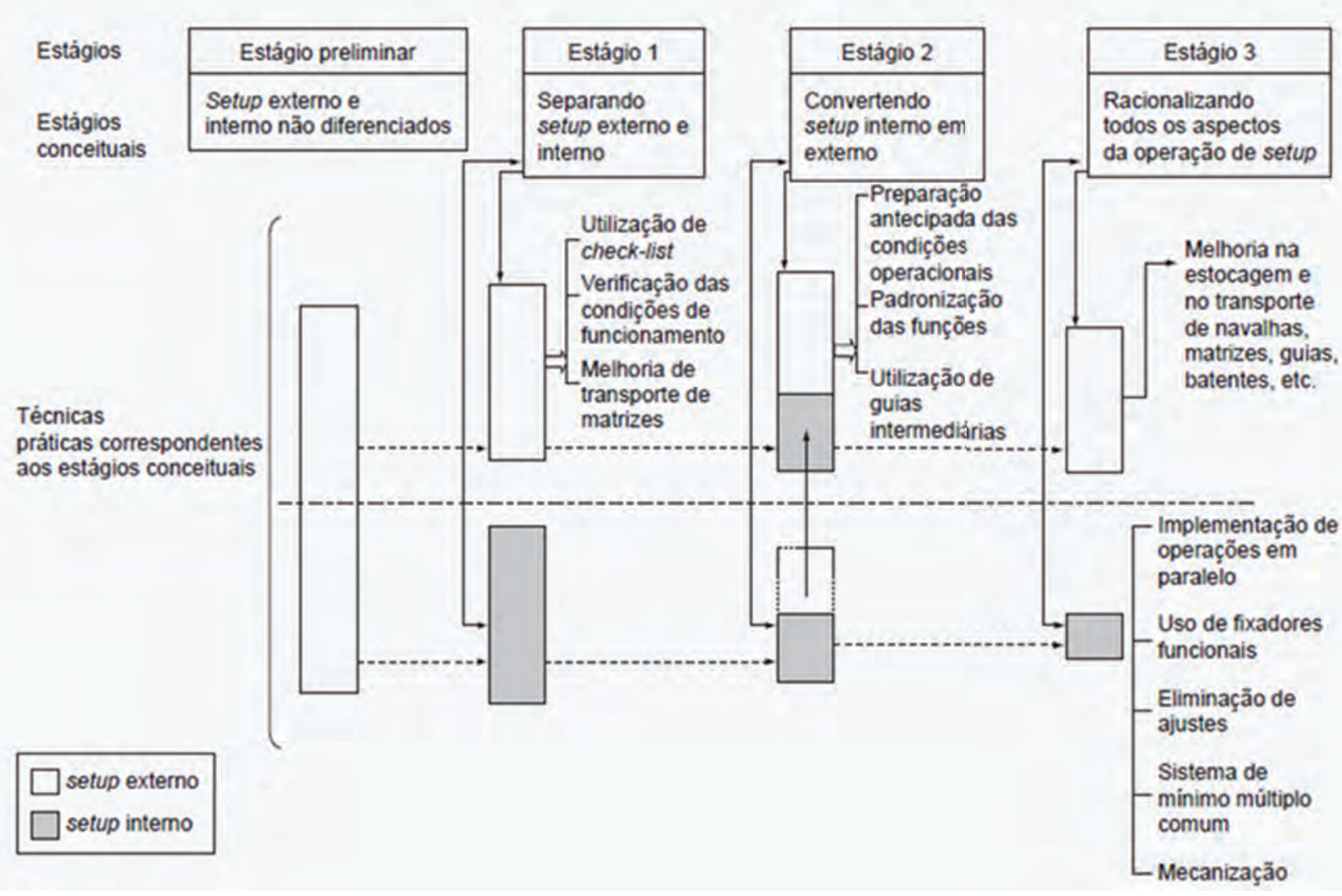

Fonte: Shingo (2000, p. 98).

Para McIntosch et al. (2007), a SMED oferece elevadas melhorias no tempo de setup a baixo custo. No entanto, os mesmos autores destacam algumas lacunas sobre a SMED:

- Não considera que pode existir uma sequência de lotes de peças diferentes, o que influi diretamente na preparação da linha, principalmente no setup;

- Há grande ênfase na questão de transferência das atividades internas em externas ao setup, o que pode ofuscar a necessidade das melhorias em projeto de máquinas;

- O período de desaceleração, que antecede o setup, e o período de aceleração, pós-setup, podem ter um desempenho muito aquém da meta de produção. Esta perda não é considerada na abordagem de Shingo (2000) e isso ocorre comumente em alguns tipos de linhas de produção e, portanto, não pode ser desconsiderado;
- A negligência durante o estágio de preparação na SMED, como a falta de reuniões periódicas e a insuficiência de treinamento dos operadores, pode comprometer o uso dessa abordagem.

Por fim, o desenvolvimento dos projetos Seis Sigma pode fazer uso da SMED, incorporando a forma com que esta evidencia de forma sistemática os tempos de paradas e de preparação dos equipamentos.

\section{Pesquisa de Campo e Análise dos Resultados}

A pesquisa foi realizada em uma multinacional situada no estado de São Paulo que atua no mercado de equipamentos de segurança e conta com cerca de 1.000 funcionários. Ela também possui o programa Seis Sigma e o Lean Production implementados. 
A empresa em estudo tem como uma das principais estratégias a redução do tempo de entrega dos seus produtos para garantir um maior nível de atendimento aos clientes. Em função disso, a situação problema identificada junto aos gestores e outros funcionários foi o elevado tempo de setup que estava contribuindo para o aumento do tempo de entrega dos produtos.

Essa situação problema encontra-se no cenário da prática do Seis Sigma e do Lean, o que permite que a investigação do desenvolvimento de um projeto Seis Sigma associado à SMED seja oportuna para que se possa aumentar, no âmbito da prática e da academia, a compreensão da integração entre o Seis Sigma a SMED durante o desenvolvimento do projeto Seis Sigma pelas fases do DMAIC.

A redução do tempo de setup no cenário do Seis Sigma e da SMED possuem o contexto e o propósito que, segundo Coughlan e Coghlan (2002), são condições para se iniciar uma pesquisa-ação na busca da solução de uma problemática vivida por gestores, contribuindo, simultaneamente, para o conhecimento (COUGHLAN; COGHLAN, 2002).

A pesquisa-ação, segundo Thiollent (1984) e Trip (2005), é um método de pesquisa caracterizado pelo envolvimento do pesquisador com a prática sem perder o embasamento teórico na busca por soluções.

A implementação da pesquisa-ação, depois de definidos o contexto e propósito, pode ser conduzida, segundo Coughlan e Coghlan (2002), em seis etapas: coleta de dados; realimentação dos dados; análise dos dados; planejamento das ações; implementação; e avaliação. Essas etapas podem ser adequadas, em termos de conteúdo e objetivo, às etapas do método DMAIC (Define, Measure, Analyse, Improve e Control) do Seis Sigma.

Na Figura 2, pode-se observar a metodologia utilizada para integrar o desenvolvimento da pesquisa pelas etapas do DMAIC às demandas da técnica SMED. Além disso, durante a execução das etapas do DMAIC destacam-se os fatores que podem influenciar a integração dessas abordagens.

Destaca-se que os envolvidos na pesquisa foram um gerente (champion), um black belt, dois engenheiros (entre eles um dos pesquisadores), os operadores de produção e um supervisor de produção (green belt).

A seguir descreve-se o desenvolvimento do projeto de redução de tempo de setup mediante o uso da SMED para cada fase do DMAIC - conforme metodologia já descrita na Figura 2.
Figura 2 - Etapas de desenvolvimento da pesquisa.

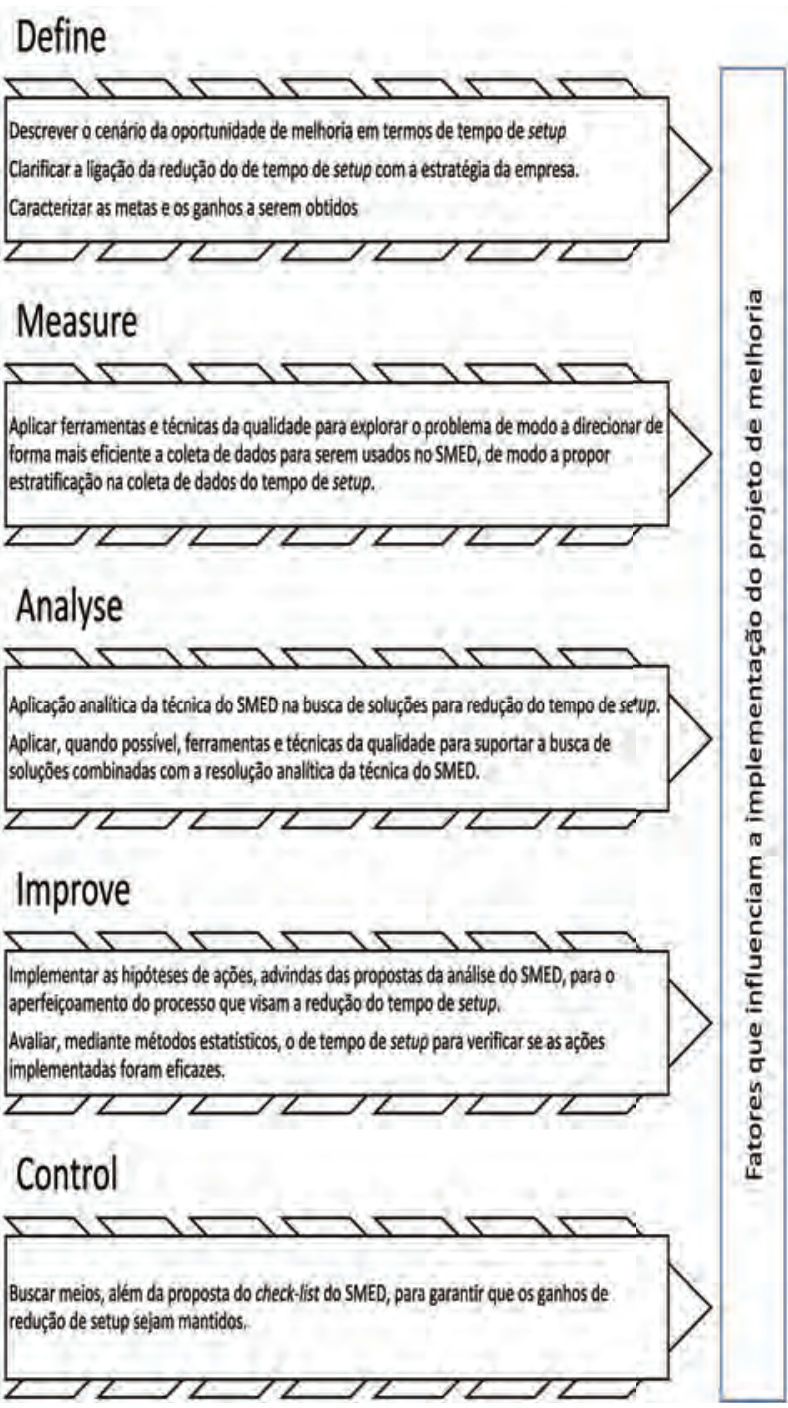

Fonte: Elaborada pelos autores.

\subsection{Define}

A máquina de conversão de filmes faz parte de uma etapa comum dos processos de fabricação das centenas de produtos da empresa. Ela já opera em três turnos com sua capacidade produtiva máxima e, em uma análise recente do processo produtivo, essa máquina foi apontada como gargalo do processo de fabricação de diversos produtos. Ao lado disso, essa máquina sofre mudanças frequentes de ferramental, cerca de três setups diários, que resultam em aproximados 90 setups mensais. Em função de a empresa ter por objetivo aproveitar os recursos da forma mais eficiente possível, os três setups diários estavam dentro da meta desejada. Os setups realizados envolvem os mesmos componentes, independente do produto a ser fabricado, variando-se apenas algumas especificações dos componentes da 
máquina. Logo, em função da problemática apresentada no início dessa seção, a escolha da máquina de conversão de filmes como objeto de aplicação do DMAIC apresentou-se coerente com a estratégia da empresa.

Durante a coleta, foi observado que os operadores e supervisores de produção já estavam preocupados sobre como as implicações do projeto de melhoria afetariam a rotina deles. Além disso, ao longo do levantamento dessas informações preliminares foram encontradas dificuldades na obtenção de dados antecessores ao início do estudo, o que conduziu a coleta de dados in loco sobre os tempos de setup.
O passo seguinte foi levantar o tempo médio de setup da máquina de conversão de filmes escolhida, que resultou em 53 minutos a partir de 50 setups, conforme Figura 3 - destaca-se que os componentes alterados durante os setups não têm influência no tempo de setup no que se refere ao tipo de componente, já que mudam apenas em termos de especificações e não de forma e peso. A partir disso, a meta foi estipulada junto ao Belts em 43 minutos, sendo o counterbalance (aquilo que não deve ser alterado devido às melhorias implementadas pelo projeto) as dimensões da margem do filme de polipropileno. Em termos mensais, considerando-se 90 setups/mês, o ganho de tempo ao se atingir a meta pode chegar a 900 minutos que equivale a $15 \mathrm{~h}$ ininterruptas de trabalho.

Figura 3 - Gráfico do histórico de tempos de setup.

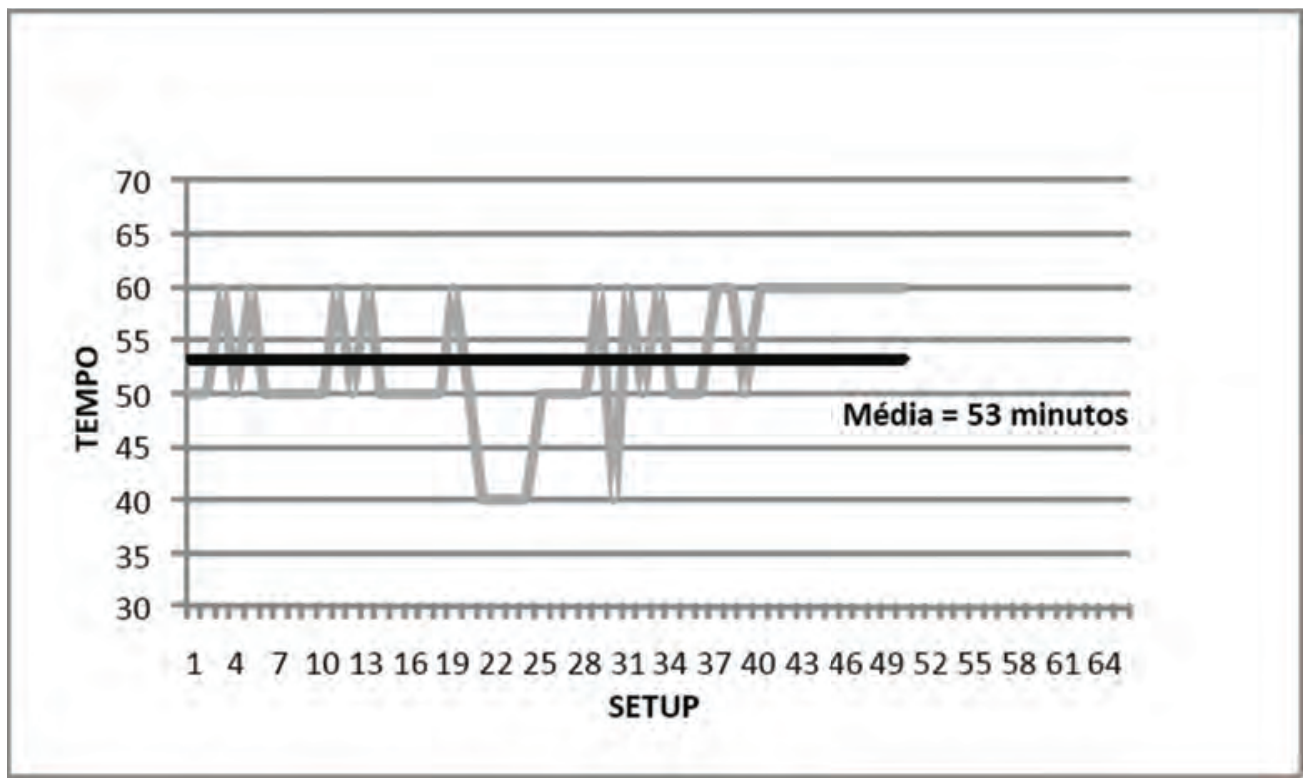

Fonte: Elaborada pelos autores.

Cabe destacar que os dados da Figura 3 foram coletados pelos operadores de produção e assumem apenas os valores 40, 50 e 60. Isso caracteriza uma imprecisão no processo de coleta de dados, que foi corrigida com treinamento e estabelecimento de uma folha de verificação adequada. Porém, isso não alterou a determinação do tempo médio em 53 minutos.

Na definição da equipe de projeto, conforme descrito anteriormente, foi feita uma abordagem individual entre os operadores dos três turnos e percebeu-se que os funcionários com mais de 10 anos de casa, demostraram desinteresse no projeto, enquanto que os mais recentes na empresa, menos de 5 anos, mostraram interesse. Não se percebeu evidências sobre o interesse (ou falta) entre os operadores entre 5 e 10 anos de casa. O turno que menos apresentou desinteresse foi o noturno devido a ser composto por funcionários com menos tempo de casa. Esse sinal de resistência é destacado por Eckes (2003), conforme o fator “mudança cultural”. Segundo esse mesmo autor, a mudança pode ir contra o que os membros da organização acreditam ser o correto e a melhor forma de lidar com isso é investir na informação, educação, treinamento e envolvimento das pessoas. Em relação aos gerentes e engenheiros, a atitude foi de elevado interesse pela condução do projeto.

Na fase Define podem ser destacados os seguintes aspectos: dificuldades de obtenção de dados antecessores; imprecisão no processo de coleta de dados in loco; e desinteresse no projeto por parte dos operadores. Esses três aspectos parecem estar relacionados ao fator mudança cultural na medida em que a falta de informação e comunicação como parte da cultura da empresa podem prejudicar o envolvimento dos operadores na coleta de dados e no desenvolvimento do projeto. 


\subsection{Measure}

Após o levantamento do histórico de tempo de setup foi elaborado o mapa do processo, utilizando as principais entradas, etapas do processo e saídas.

A partir disso elaborou-se uma matriz de causa-e-efeito, na qual foram identificadas via brainstorm as variáveis independentes do processo (Xs), que foram analisadas via o Diagrama de Pareto da Figura 4. A pontuação foi obtida mediante a atribuição de notas $(0,1,3$ ou 9, em que 0 não tem relação e 9 tem elevada relação) ponderando o quanto “ $X$ ” contribuiria para redução da variável dependente (Y), tempo de setup. Essa ponderação também levou em consideração o quanto $\mathrm{X}$ afetaria negativamente as dimensões da margem do filme de polipropileno.

Com base no Pareto da Figura 4 foram selecionados os nove primeiros " $X$ ", que representam mais de $80 \%$ dos fatores que afetam o tempo de setup para se aplicar a ferramenta FMEA (Failure Modes Effects and Analysis).

No FMEA atribuiu-se pontuação levando em conta o efeito da falha, frequência que ocorre e controle atual de cada um dos nove " $\mathrm{X}$ ” selecionados anteriormente. Os dados necessários foram obtidos no banco de dados da empresa e, apesar do questionamento sobre sua confiabilidade, foram utilizadas no desenvolvimento do novo Pareto da Figura 5.
A aplicação do FMEA apontou os " $X$ " que deveriam ser priorizados mediante a análise de Pareto para se atingir o objetivo de redução do tempo de setup. A seguir uma análise sobre cada X é efetuada.

Figura 4 - Gráfico de Pareto.

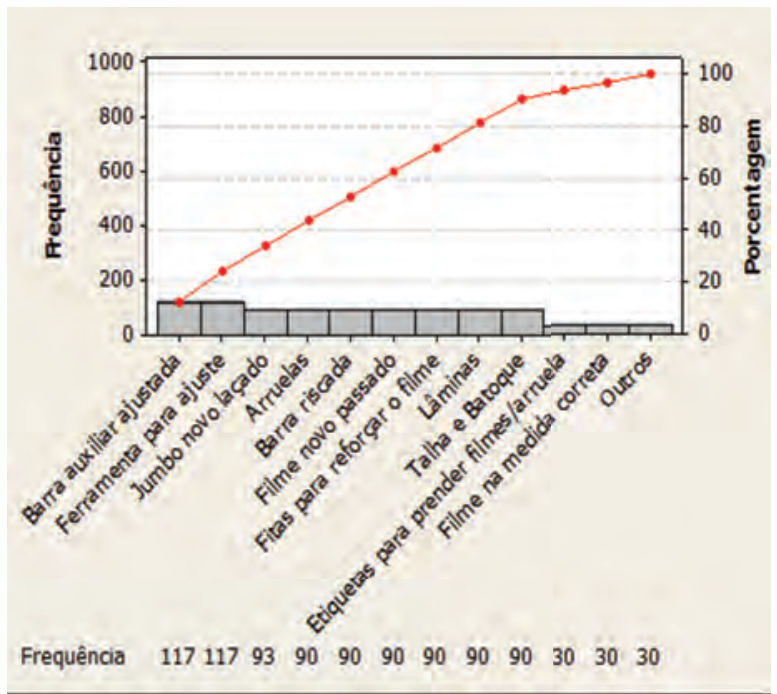

Fonte: Elaborada pelos autores.

Um fator destacado como crítico nessa fase foi a necessidade de treinamento para fortalecer o entendimento e aplicação das ferramentas durante a realização da fase. A “educação e treinamento contínuos” é um dos fatores destacado por Antony e Banuelas (2002).

Figura 5 - Pareto sobre FMEA.

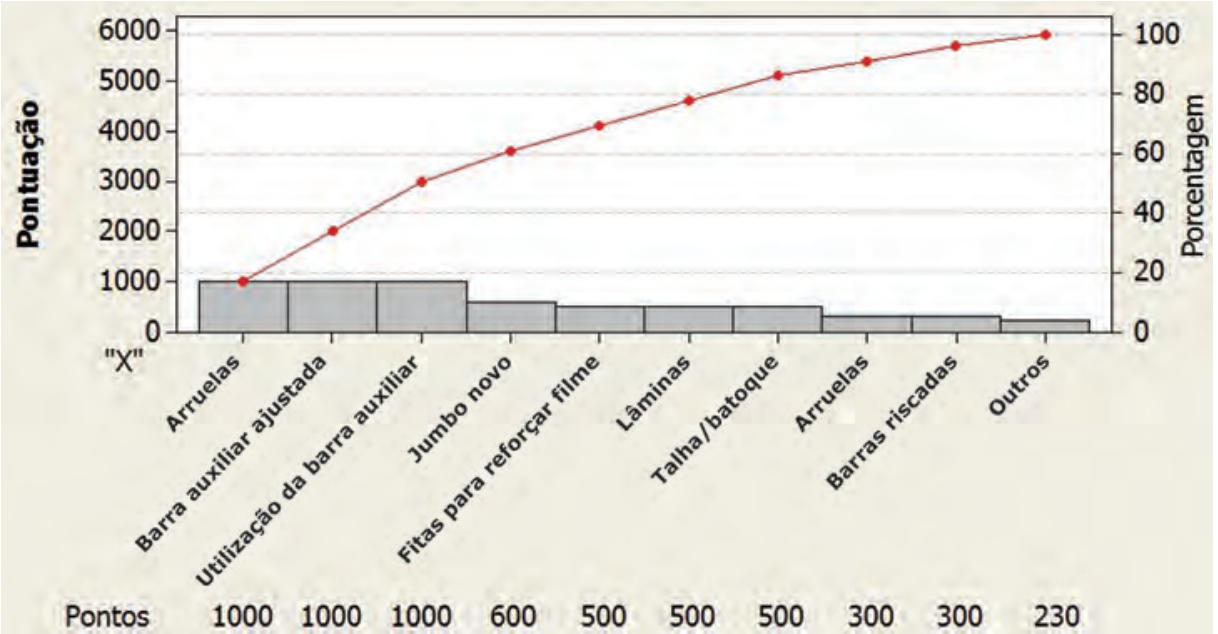

Fonte: Elaborada pelos autores.

Na fase Measure podem ser destacados os seguintes aspectos: necessidade de treinamento contínuo; e desconfiança dos dados. Esses aspectos estão relacionados aos fatores “educação e treinamento contínuos” e “mudança cultural”. Nota-se que o segundo pode ser destacado como prejudicial ao desenvolvimento do projeto, pois está associado com a desconfiança dos dados utilizados na identificação de algumas variáveis independentes. Mais uma vez a barreira apontada por Eckes (2003) parece afetar negativamente o desenvolvimento do projeto. 


\subsection{Analyse}

Considerando o Pareto da Figura 5 procedeu-se com a aplicação da SMED. Inicialmente, foi realizada a classificação do setup em interno e externo, dentro do cenário atual (ATUALMENTE), após a aplicação da SMED ideal (APÓS SMED) e o com a aplicação da SMED dentro do possível (POSSÍVEL) - Quadro 2.

A partir do Quadro 2, é possível perceber que o uso da "barra auxiliar" contribui expressivamente para a redução do tempo total de setup, ao passo que a utilização de mais "batoques”, que implicaria num investimento elevado, não reduz de maneira expressiva a redução total de setup.
Outras atividades que entraram na análise, além das previstas no FMEA, e resultaram na melhoria do tempo de setup foram "olhar standard de produção" e "preencher relatório”.

Ainda em relação ao Quadro 2, percebe-se que considerando as melhorias possíveis de serem aplicadas (POSSÍVEL), o tempo de setup poderia ser reduzido para 28 minutos, o que reduziria o tempo médio de setup em $47,2 \%$.

Foi elaborado um check-list de setup para organizar, controlar e garantir que as atividades de setup externo fossem realizadas, conforme recomendações de Shingo (2000).

Quadro 2 - Análise SMED.

\begin{tabular}{|c|c|c|c|c|c|c|c|}
\hline \multicolumn{2}{|c|}{} & \multicolumn{2}{c|}{ ATUALMENTE } & \multicolumn{2}{c|}{ APÓS SMED } & \multicolumn{2}{c|}{ POSSÍVEL } \\
\hline ATIVIDADE & Tempo & Interno & Externo & Interno & Externo & Interno & Externo \\
\hline Retirar canela usada & 2 & & & & & & \\
\hline Retirar Batoque & 2 & & & & & & \\
\hline Laçar Jumbo & 3 & & & & & & \\
\hline Colocar batoque & 2 & & & & & & \\
\hline Colocar Jumbo & 3 & & & & & & \\
\hline Emenda de filme & 2 & & & & & & \\
\hline Preparação da Barra & 10 & & & & & & \\
\hline Troca de barra* & 1 & & & & & & \\
\hline Passagem do filme & 6 & & & & & & \\
\hline Selecionar Arruelas & 3 & & & & & & \\
\hline Ajuste de arruelas & 7 & & & & & & \\
\hline Retirar material das barras & 3 & & & & & & \\
\hline Olhar Standard* & 4 & & & & & & \\
\hline Preencher Relatório* & 3 & & & & & & \\
\hline TOTAL & 51 & & 51 & & 26 & & 28 \\
\hline
\end{tabular}

Fonte: Elaborado pelos autores.

Durante a fase Analyse a interação com todos os envolvidos no projeto (operadores, engenheiros e gerentes) foi marcante, principalmente com os operadores do turno noturno. Um destaque nessa fase que ajudou o desenvolvimento do projeto foi a apresentação dos resultados alcançados preliminares do DMAIC com a SMED para a gerência. Isso forneceu credibilidade ao projeto e contribuiu para convencer os engenheiros e os gerentes das soluções que deveriam ser tomadas para a fase Improve.

Os operadores também contribuíram, dando sugestões sobre a disposição de ferramentas e matéria-prima no local de produção e a falta de insumos, o que não foi observado nas fases anteriores.

Na fase Analyse podem ser destacados os seguintes aspectos: uso dos resultados do Pareto e FMEA para auxiliar na classificação dos setups internos e externos (POSSÍVEL); e aumento da credibilidade dos possíveis ganhos por parte dos gerentes, engenheiros e até operadores. Esses aspectos estão relacionados ao fator "compreensão do programa Seis Sigma”, pois a divulgação da análise mediante a sistemática clara e concisa do DMAIC proporcionou credibilidade a forma como o projeto estava sendo conduzido. 


\subsection{Improve}

Nessa etapa ocorreu a implementação das melhorias identificadas na fase anterior.

As melhorias constituíram-se na implementação das mudanças de setup interno para externo, identificadas na aplicação da SMED da etapa anterior - Quadro 2.
Na Figura 6 é apresentado o tempo médio de setup antes e depois da implementação da melhoria. Nota-se que a média foi reduzida de 53 minutos para 45 minutos, ficando acima da meta de 43 minutos. Em termos mensais, considerando-se 90 setups/mês, o ganho de tempo ao se atingir a meta pode chegar a 720 minutos que equivale a $12 \mathrm{~h}$ ininterruptas de trabalho.

Figura 6 - Melhoria do tempo de setup antes vs. depois.

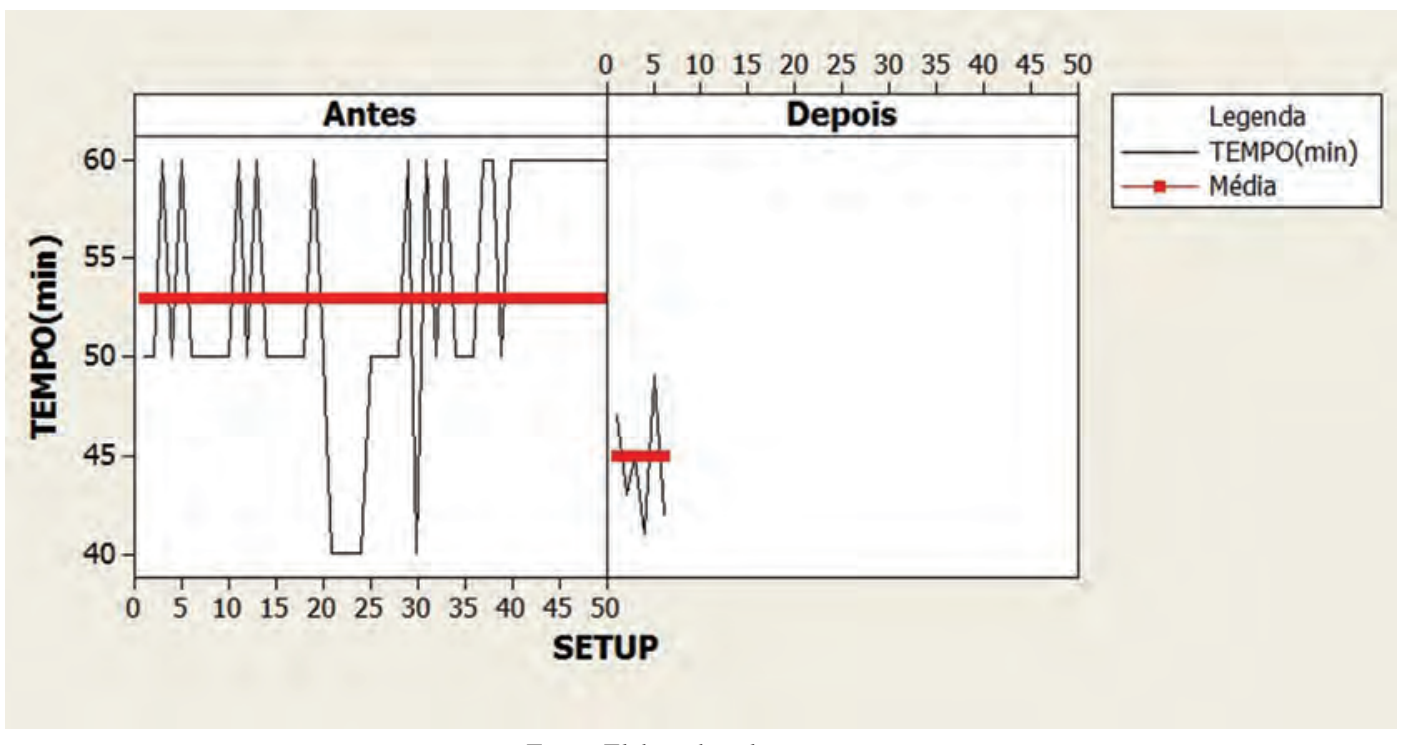

Fonte: Elaborada pelos autores.

Para confirmar os ganhos obtidos em termos estatísticos foram aplicados testes de hipótese para média e para variância, segundo Montgomery e Runger (2009). O primeiro teste foi para duas médias não-emparelhadas com desvio-padrão da população desconhecido, teste “t” e outro para duas variâncias, teste " $F$ ”. Os resultados dos testes são apresentados no Quadro 3 e são analisados para um nível de significância igual a 5\%.

De acordo com os resultados do Quadro 3, tem-se que para média o p-valor $=0,000$ e é inferior a 0,05, o que indica que existem evidências estatísticas para se rejeitar $\mathrm{H}_{0}$, ou seja, que a média do processo mudou. Já para variância, como o p-valor $=0,201$ é superior a 0,05 , não existem evidências para se rejeitar $\mathrm{H}_{0}$, ou seja, tudo indica que a variância, que está associada a dispersão dos valores, não mudou para um nível de 5\% de significância.

Quadro 3 - Testes de Hipótese.

\begin{tabular}{|c|l|c|c|}
\hline & \multicolumn{1}{|c|}{ Hipótese } & p-valor & Resultado \\
\hline \multirow{2}{*}{ Média } & $\mathrm{H}_{0}=$ médias iguais & \multirow{2}{*}{0,000} & \multirow{2}{*}{ Rejeita-se $\mathrm{H}_{0}$} \\
\cline { 2 - 2 } & $\mathrm{H}_{1}=$ médias diferentes & & \\
\hline \multirow{2}{*}{ Variância } & $\mathrm{H}_{0}=$ variâncias iguais & \multirow{2}{*}{0,201} & \multirow{2}{*}{ Não rejeita-se $\mathrm{H}_{0}$} \\
\cline { 2 - 2 } & $\mathrm{H}_{1}=$ variâncias diferentes & & \\
\hline
\end{tabular}

Fonte: Elaborado pelos autores.
Durante a implementação das melhorias, iniciou-se um processo de aproximação com os operadores, por meio de conversas explicativas dos benefícios das melhorias. Mesmo assim, foi percebida, mediante reclamações, dificuldade dos operadores em mudar a rotina das atividades deles em função da nova dinâmica de trabalho. Especificamente, os turnos da manhã e da tarde, compostos em sua maioria por funcionários com mais de 10 anos de casa, foram os que geraram maior resistência às mudanças.

Na fase Improve podem ser destacados os seguintes aspectos: uso da estatística para corroboração dos resultados; e resistência à mudança por parte dos operadores. Esses aspectos estão relacionados aos fatores “compreensão do programa Seis Sigma” e “mudança cultural”. Mais uma vez a mudança cultural mostrou-se como crítica para o desenvolvimento de um projeto de melhoria para essa organização.

\subsection{Control}

Para controlar os tempos de setup obtidos com a melhoria do processo foi implantado um quadro de controle visual próximo à máquina. Essa recomendação está na proposta de Pande et al. (2000). 
O quadro de controle visual constituía-se no uso de cartões verdes, amarelos e vermelhos, que abrangem os 10 últimos setups. Se mais de $90 \%$ dos setups atingissem a meta, então a máquina recebia um cartão da cor verde, caso a quantidade dos últimos dez setups ficasse entre $60 \%$ e $90 \%$ da meta, colocava-se um cartão amarelo e em caso de menos de $60 \%$ dos casos atingirem a meta, utilizava-se um cartão vermelho. Destaca-se que a escala determinada foi colocada apenas como referência para que os operadores fossem incentivados a realizar o setup dentro da meta.

De acordo com observações diretas e indiretas, notou-se que a instalação do quadro de controle visual gerou desconforto entre os operadores. Eles se sentiram vigiados e controlados, segundo relatos coletados em campo. Além disso, o impacto negativo foi ainda maior nos operadores dos turnos da manhã e da tarde. Por outro lado, a aceitação por parte da gerência e dos engenheiros foi elevada, pois a partir dessa iniciativa, o status dos setups passou a ser obtido de forma rápida.

Na fase Control podem ser destacados os seguintes aspectos: “mudança cultural”. Este fator também está agindo como prejudicial ao desenvolvimento do projeto.

\section{Considerações finais}

A investigação da aplicação do DMAIC com a SMED evidenciou que essa combinação pode trazer benefícios, porém algumas dificuldades foram constadas.

A pesquisa mostrou que o Seis Sigma forneceu uma infraestrutura de apoio ao desenvolvimento da SMED. Inicialmente, o método DMAIC forneceu orientação estratégica na aplicação da SMED. Outro destaque foi para a estrutura de belts, que em conjunto com as ferramentas da qualidade e os métodos estatísticos, conduziram a uma resolução sistemática do problema de redução de tempo de setup. Esses elementos aumentam a chance de sucesso de projetos dessa natureza.

Durante o desenvolvimento do DMAIC foi possível observar que alguns dos fatores levantados na revisão da literatura influenciaram o desenvolvimento do projeto Seis Sigma. Entre eles, os destacados foram: mudança cultural, educação e treinamento contínuos, e compreensão do programa Seis Sigma.

O fator "mudança cultural” estava associado à falta de confiabilidade nas medidas coletadas, falta de comunicação no início do projeto sobre seu impacto no dia-a-dia dos operários e resistência à mudança entre os operadores. Uma forma de amenizar o efeito negativo desse fator seria investir em treinamentos que enfatizem a importância dos fatos e dados no processo de tomada de decisão.
O fator “educação e treinamento contínuos” foi marcante nas fases Measure e Analyse devido às exigências dessas fases quanto ao uso de ferramentas da qualidade e métodos estatísticos do Seis Sigma. Isso requer que os treinamentos não sejam pontuais, mas contínuos de forma que as pessoas estejam sempre seguras para desenvolver novas soluções a partir de novos conhecimentos, como foi o caso do uso da SMED.

O fator “compreensão do programa Seis Sigma” na fase Analyse proporcionou maior entendimento entre os envolvidos por meio da apresentação baseada em fatos e dados dos resultados esperados. Isso, até certo ponto, amenizou os resultados negativos percebidos pelo fator "mudança cultural”. Os ganhos desse fator estão associados ao aumento do comprometimento da alta administração e até do operacional para com o programa Seis Sigma.

De modo geral, a resistência à mudança foi a principal barreira na aplicação do DMAIC com a SMED. Isso aponta que esses métodos precisam de preparação antes da aplicação, pois além das soluções técnicas, é necessário que o comprometimento de todos, inclusive na manutenção das melhorias depois de implementada a solução técnica.

Destaca-se que essa pesquisa, apesar das restrições oriundas do método da pesquisa-ação, contribuiu de forma incremental ao conhecimento, conforme destaque de Coughlan e Coghlan (2002) sobre o método. O incremento ao conhecimento foi em relação ao entendimento da integração entre o DMAIC do Seis Sigma e a técnica SMED, que quando combinados podem ter seus resultados potencializados.

Uma das limitações do trabalho está na etapa de controle que, devido às restrições de tempo do pesquisador, não pôde ter o acompanhamento continuado para que mais dados pudessem ser analisados para fornecer informações sobre a estabilidade da melhoria, com isso é conveniente considerar que ganho de tempo alcançado seja entendido como potencial e não real.

\section{Referências}

ANTONY, J.; BANUELAS, R. Key ingredients for the effective implementation of Six Sigma program. Measuring Business Excellence, v. 6, n. 4, p. 20-27, 2002.

ARIENTE, M.; CASADEI, M. A. GIULIANI, A. C.; SPERS, E. E. Processo de mudança organizacional: estudo de caso do Seis Sigma. Revista FAE, Curitiba, v. 8, n. 1, p. 81-92, 2005. 
ARNHEITER, Edward D.; MALEYEFF, John. The integration of lean management and Six Sigma. The TQM magazine, v. 17, n. 1, p. 5-18, 2005.

ATMACA, E.; GIRENES, S.S. Lean Six Sigma methodology and application. Quality \& Quantity, v. 47, n. 4, p. 2107-2127, 2013.

BEN NAYLOR, J.; NAIM, Mohamed M.; BERRY, Danny. Leagility: integrating the lean and agile manufacturing paradigms in the total supply chain. International Journal of Production Economics, v. 62, n. 1, p. 107-118, 1999.

BENNER, M. J.; VELOSO, F. M. ISO 9000 practices and financial performance: A technology coherence perspective. Journal of Operations Management, v. 26, n. 5, p. 611-629, set. 2008.

BRAUNSCHEIDEL, M. J.; HAMISTER, J. W.; SURESH, N. C.; STAR, H. An institutional theory perspective on Six Sigma adoption. International Journal of Operations \& Production Management. v. 31, n. 4, p. 423-451, 2011.

CAKMAKCI, M. Process improvement: performance analysis of the setup time reduction-SMED in the automobile industry. International Journal of Advanced Manufacturing Technology, v. 41, p. 168-179, 2009.

CHEN, C. Y. CHANG, P. Y. Implementation of the Lean Six Sigma framework in non-profit organisations: A case study. Total Quality Management \& Business Excellence, v. 23, n. 4, p. 431-447, 2012.

CORONADO, R. B.; ANTONY, J. Critical success factors for the successful implementation of Six Sigma projects in organisations. The TQM Magazine, v. 14, n. 2, p. 92-99, 2002.

COUGHLAN, P.; COGHLAN, D. Action research for operations management. International Journal of Operations \& Production Management, v. 22, n. 2, p. 220-240, 2002.

ECKES, George. Six Sigma for everyone. Wiley, 2003.

FOLARON, J. The Evolution of Six Sigma. Six Sigma Forum Magazine, v. 2, n. 4, p. 35-45, 2003.

JOHNSON, A., SWISHER, B. How six sigma improves R\&D. Research Technology Management, v. 46, n. 2, p. 12-15, 2003.

KARASU, M. K.; CAKMAKCI, M.; CAKIROGLU, M. B.; AYYA, E.; DEMIREL-ORTABAS, N. D. Improvement of changeover times via Taguchi empowered SMED/case study on injection molding production. Measurement, v. 47, p. 741-748, 2014.
KWAK, Y.; ANBARI, F. Success Factors in Managing Six Sigma Projects. In: Project Management Institute Research Conference, UK, July. 2004.

McINTOSH, R. I.; SUGAI. M.; NOVASKI. O. Metodologia de Shigeo Shingo (SMED): análise crítica e estudo de caso. Gestão e Produção, v. 14, n. 2, p. 323-335, 2007.

MONTGOMERY, D. C.; RUNGER, George C. Estatística aplicada à engenharia. Livros Técnicos e Científicos, 2009.

PANDE, P.S.; NEUMAN, R.P.CAVANAGH,R.R. The Six Sigma Way: How GE, Motorola, and other companies are honing their performance, p. 427, 2000.

PINHO, C.T.A. Seis Sigma: Uma proposta para implementação da metodologia em pequenas empresas. Dissertação (Mestrado) - Programa de Engenharia de Produção, Centro de Tecnologia, Universidade Federal do Rio Grande do Norte. Natal, Rio Grande do Norte, 2005. 105 p.

RODRIGUES, J.; WERNER, L. Descrevendo o Programa Seis Sigma: Uma Revisão da Literatura. In: XXVIII Encontro Nacional de Engenharia de Produção, Rio de Janeiro, RJ, Brasil, out. 2008.

SALAH, Souraj; RAHIM, Abdur; CARRETERO, Juan A. The integration of Six Sigma and lean management. International Journal of Lean Six Sigma, v. 1, n. 3, p. 249-274, 2010.

SATOLO, E.G.; ANDRIETTA, J.M.; MIGUEL; P.A.C.; CALARGE, F.A. Análise da utilização de técnicas e ferramentas no programa Seis Sigma a partir de um levantamento tipo survey. Produção, v. 19, n. 2, p. 400-416, 2009.

SCHROEDER, R. G.; LINDERMAN, K..; LIEDTKE, C.; CHOO, A. Six sigma: definition and underlying theory. Journal of Operations Management, v. 26, n. 4, p. 536-554, 2008.

SHAFER, S. M.; MOELLER, S. B. The effects of Six Sigma on corporate performance: An empirical investigation. Journal of Operations Management, v. 30, n. 7-8, p. 521-532, nov. 2012.

SHINGO, S. Sistema de Troca Rápida de Ferramenta: uma revolução nos sistemas produtivos. Bookman, Porto Alegre, 2000.

SWINK, M.; JACOBS, B. W. Six Sigma adoption: Operating performance impacts and contextual drivers of success. Journal of Operations Management, v. 30, n. 6, p. 437-453, 2012. 
THIOLLENT, M. Aspectos Qualitativos da Metodologia de Pesquisa com Objetivos de Descrição, Avaliação e Reconstrução. Simpósio, Caderno de Pesquisa, Rio de Janeiro, 1984.

TRAD, S.; MAXIMIANO, A.C.A. Seis Sigma: Fatores críticos de sucesso para sua implantação. Revista de Administração Contemporânea, Curitiba, v. 13, n. 4, p. 647-662, 2009.
TRIP, D. Pesquisa-ação: uma introdução metodológica. Educação e Pesquisa, São Paulo, v. 31, n. 3, p. 443-466, set./dez. 2005.

YUSR, M.; OTHMAN, A. R.; MOKHTAR, S. S. M. Assessing the Relationship among Six Sigma, Absorptive Capacity and Innovation Performance. Procedia - Social and Behavioral Sciences, v. 65, p. 570-578, 2012. 